\title{
Impulsive Vaccination SEIR Model with Nonlinear Incidence Rate and Time Delay
}

\author{
Dongmei Li, Chunyu Gui, and Xuefeng Luo \\ Department of Applied Mathematics, Harbin University of Science and Technology, Harbin 150080, China \\ Correspondence should be addressed to Dongmei Li; dmli_2013@126.com
}

Received 12 July 2013; Revised 22 October 2013; Accepted 30 October 2013

Academic Editor: Jun $\mathrm{Hu}$

Copyright (C) 2013 Dongmei Li et al. This is an open access article distributed under the Creative Commons Attribution License, which permits unrestricted use, distribution, and reproduction in any medium, provided the original work is properly cited.

\begin{abstract}
This paper aims to discuss the delay epidemic model with vertical transmission, constant input, and nonlinear incidence. Some sufficient conditions are given to guarantee the existence and global attractiveness of the infection-free periodic solution and the uniform persistence of the addressed model with time delay. Finally, a numerical example is given to demonstrate the effectiveness of the proposed results.
\end{abstract}

\section{Introduction}

Vaccination has been widely used as a method of disease control; inoculate is an effective approach according to the characteristics of the disease which takes the defense in advance. The implementation of inoculate is not continuous but cyclical. As early as in the 1960s, the principle of the stability has been given in [1] for the impulsive differential equation. Subsequently, a better definition of stability has been proposed in [2] for the impulsive differential equation. Motivated by the above work, the attention has been made on the application of the pulse immunization in the infectious disease model. For example, the study has been reported in [3] where the research has been made about inoculating the pulse vaccination for the people who are easily infected. The researchers have realized that the pulse vaccination strategies can eliminate the positive role to measles; see for example $[3,4]$. Moreover, the SIV infection model has been studied in [5]. The inoculation ratio and the inoculation interval time to eliminate the influence of the disease have been pointed out. Accordingly, a huge amount of results has appeared concerning the pulse model to study epidemics of infectious diseases; see for example [6,7].

On the other hand, the pulse SEIR epidemic model with the incubation period has been established in [8]. The qualitative analysis has been given that the local asymptotic stability of infection-free periodic solution is globally stable. Note that the existence of time delay would degrade the desired performance or even result in the instability [9-12]. As such, a new class of models with vertical transmission delay pulse infectious disease has been given in [7]. By using the impulsive differential inequality, the sufficient conditions have been presented to guarantee the global attractability of infection-free periodic solution and uniform persistence of the disease. Subsequently, the disease delay pulse models with multiple infectious diseases have been addressed in [13] and the SIR pulse vaccination infectious disease model with time delay has been discussed in [14]. By constructing an appropriate Lyapunov function, the global attractability of the unique positive periodic solution has been discussed in [15] for the pulse predator-prey system with distributed delay and proliferated. Also, by using the comparison principle of impulsive differential equations, the influence from the time delay, the pulse vaccination, and the other factors on the nature of the model has been tackled in $[16,17]$.

An impulsive vaccination SEIR epidemic model with saturation infectious and constant input has been studied in [18], and the sufficient conditions have been established to ensure the stability and the persistence of the disease-free periodic solution. In [19], the SEIRS pulse infectious disease model with saturated incidence has been investigated. Sufficient conditions of infection-free periodic solution and diseaselasting conclusion have been obtained. Also, it has been shown that the size of the pulse cycle is an important factor affecting the disease extinction. The SIR epidemic model with nonlinear incidence rate and two class infectious diseases 
containing the pulse effect has been studied in [20]. Sufficient conditions of the global attractability of the disease-free periodic solutions have been given. It has confirmed that the system is uniformly persistent under a certain condition. Meanwhile, the time delay, pulse vaccination, and nonlinear incidence play important roles in the nature of the model. The conditions have been proposed to control two kinds of diseases. In [21], the pulse vaccination SIQRS epidemic model with constant input and saturated incidence rate has been addressed. However, it is worth mentioning that the unified model with vertical transmission, constant input, and nonlinear incidence has not been investigated.

In this paper, the cases of constant population input and vertical transmission are considered. Patients who contact susceptible people with saturated incidence way are taken into account. A new impulsive vaccination SEIR epidemic model with time delay and nonlinear incidence rate is established. The sufficient condition is given to guarantee the stability of the disease-free periodic solution and the persistence of the model. Compared to existing results, the main contributions lie in the following aspects: (i) the nonlinear incidence rate is considered in the model to describe the spread of the disease which is more close to reality; (ii) all kinds of infectious diseases have the incubation period, and therefore it is necessary to deal with the phenomenon of time delay; (iii) a unified pulse SEIR epidemic model including the nonlinear contract rate, vertical transmission, and time delays is established. As discussed in [22-24], the study of the pulse epidemic model conducted in this paper has analyzed the trend of the disease in the theoretical aspect which will contribute to making the strategy of the disease prevention.

\section{Model Establishment}

We consider an SEIR model by assuming that the input term has a constant population, a nonlinear occurrence rate as $\beta S(t) I(t) /\left(1+m I^{h}(t)\right)$, and the number of sick of which the sick people who birth to the newborn are $q \mu I(t)$, the time needed which the lurker transfer into the infected people is $\tau$, so after a time $\tau$, the number of survived lurker which into the infected person is

$$
\frac{\beta S(t-\tau) I(t-\tau)}{1+m I^{h}(t-\tau)} e^{-\mu \tau}+q \mu I(t-\tau) e^{-\mu \tau} .
$$

The effective coverage of the pulse vaccination needle for the newborn that is not infected is denoted by $\theta$; the vaccination cycle is $T$. Now we can obtain the following impulsive differential equation model:

$$
\begin{gathered}
S^{\prime}(t)=A-\frac{\beta S(t) I(t)}{1+m I^{h}(t)}-\mu S(t)-q \mu I(t), \\
E^{\prime}(t)=\frac{\beta S(t) I(t)}{1+m I^{h}(t)}-\frac{\beta S(t-\tau) I(t-\tau)}{1+m I^{h}(t-\tau)} e^{-\mu \tau}+q \mu I(t) \\
-q \mu I(t-\tau) e^{-\mu \tau}-\mu E(t), \\
I^{\prime}(t)=\frac{\beta S(t-\tau) I(t-\tau)}{1+m I^{h}(t-\tau)} e^{-\mu \tau}+q \mu I(t-\tau) e^{-\mu \tau} \\
-(\mu+\gamma+\alpha) I(t),
\end{gathered}
$$

$$
\begin{gathered}
R^{\prime}(t)=\gamma I(t)-\mu R(t), \\
t \neq n T, \\
S\left(t^{+}\right)=S(t)-\theta \mu(S(t)+E(t)+R(t))-\theta p \mu I(t), \\
E\left(t^{+}\right)=E(t), \\
I\left(t^{+}\right)=I(t), \\
R\left(t^{+}\right)=R(t)+\theta \mu(S(t)+E(t)+R(t))+\theta p \mu I(t), \\
t=n T .
\end{gathered}
$$

Here, $S(t), E(t), I(t)$, and $R(t)$ represent susceptible, lurker, disease, and cure at time $t$, respectively; $\mu$ represents the birth rate (the birth rate is equal to death rate); $\beta$ represents the effective contact number; $\alpha$ is the mortality due to illness; $\gamma$ denotes the cure rate; $q(0<q<1)$ represents the infected people who give birth to the newborn and who are vertically infected into "the kind of latent" at time $t ; p=1-q$ is the proportion of infected people who gave birth to newborn who are not vertically infected at time $t ; \theta(0<\theta<1)$ denotes the succeeded vaccination proportion of newborn for all those who are not infected; $A$ represents input number of the population of constant; $\tau$ denotes the time of lurker who become infected people; T is the pulse vaccination cycle; $h, m$, $A, \mu, \beta$, and $\gamma$ are positive constants. In this paper, we consider the property of the model under $S(t) \geq 0, E(t) \geq 0, I(t) \geq 0$, and $R(t) \geq 0$, and the initial conditions are given as follows:

$$
\begin{gathered}
\left(\varphi_{1}(s), \varphi_{2}(s), \varphi_{3}(s), \varphi_{4}(s)\right) \in C\left([-\tau, 0], R_{+}^{4}\right), \\
\varphi_{i}(0)>0 \quad(i=1,2,3,4) .
\end{gathered}
$$

Letting $N(t)=S(t)+E(t)+I(t)+R(t)$ and according to (2), we have

$$
\begin{aligned}
N^{\prime}(t) & =S^{\prime}(t)+E^{\prime}(t)+I^{\prime}(t)+R^{\prime}(t) \\
& =A-\mu N(t)-\alpha I(t) .
\end{aligned}
$$

So (2) can be transformed into the following form:

$$
\begin{gathered}
S^{\prime}(t)=A-\frac{\beta S(t) I(t)}{1+m I^{h}(t)}-\mu S(t)-q \mu I(t), \\
E^{\prime}(t)=\frac{\beta S(t) I(t)}{1+m I^{h}(t)}-\frac{\beta S(t-\tau) I(t-\tau)}{1+m I^{h}(t-\tau)} e^{-\mu \tau}+q \mu I(t) \\
-q \mu I(t-\tau) e^{-\mu \tau}-\mu E(t), \\
I^{\prime}(t)=\frac{\beta S(t-\tau) I(t-\tau)}{1+m I^{h}(t-\tau)} e^{-\mu \tau}+q \mu I(t-\tau) e^{-\mu \tau} \\
-(\mu+\gamma+\alpha) I(t), \\
N^{\prime}(t)=A-\mu N(t)-\alpha I(t), \\
S\left(t^{+}\right)=S(t)-\theta \mu N(t)+\theta \mu q I(t), \\
E\left(t^{+}\right)=E(t), \\
I\left(t^{+}\right)=I(t), \\
N\left(t^{+}\right)=N(t), \\
t=n T .
\end{gathered}
$$


Noticing $N^{\prime}(t) \leq A-\mu N(t)$ and by the comparison principle, we have $N^{\prime}(t) \leq(A / \mu)+e^{-\mu t}(N(0)-(A / \mu))$ and then we have $\lim _{t \rightarrow \infty} N(t) \leq(A / \mu)$. Then, all solutions $(S(t), E(t), I(t)$, and $N(t))$ of model (5) eventually enter and remain in the domain $\Omega=\left\{(S, E, I, N) \in R_{+}^{4}: N \leq A / \mu\right\}$. Therefore, $\Omega$ is the positive invariant set of (5).

To proceed, we introduce the following two lemmas which will play important roles in the remaining parts of this paper.

Lemma 1 (see [25]). Consider the following differential equations with delay:

$$
\begin{gathered}
w^{\prime}(t) \leq(\geq) p(t) w(t)+q(t), \quad t \neq t_{k}, \\
w\left(t_{k}^{+}\right) \leq(\geq) d_{k} w\left(t_{k}\right)+b_{k}, \quad t=t_{k}, k \in N,
\end{gathered}
$$

where $p(t), q(t) \in C\left[R_{+}, R\right], d_{k} \geq 0$, and $b_{k}$ are constants. Assume that

(i) the sequence $\left\{t_{k}\right\}$ satisfies $0 \leq t_{1}<t_{2}$ and $\lim _{t \rightarrow \infty} t_{k}=$ $\infty$;

(ii) $w \in P C^{\prime}\left[R_{+}, R\right]$ and $w(t)$ are left continuous at $t_{k}(k \in$ $N)$, then

$$
\begin{aligned}
w(t) \leq & (\geq) w\left(t_{0}\right) \prod_{t_{0}<t_{k}<t} d_{k} \exp \left(\int_{t_{0}}^{t} p(s) d s\right) \\
& +\sum_{t_{0}<t_{k}<t}\left(\prod_{t_{k}<t_{j}<t} d_{j} \exp \left(\int_{t_{k}}^{t} p(s) d s\right)\right) b_{k} \\
& +\int_{t_{0}}^{t} \prod_{s<t_{k}<t} d_{k} \exp \left(\int_{s}^{t} p(\theta) d \theta\right) q(s) d s, \quad t \geq t_{0} .
\end{aligned}
$$

Lemma 2 (see [26]). Considering the following differential equations with delay:

$$
x^{\prime}(t)=r_{1} x(t-\tau)-r_{2} x(t),
$$

where $r_{1}, r_{2}$, and $\tau$ are positive constants, and $x(t)>0$, one has the following

(i) if $r_{1}<r_{2}$, then $\lim _{t \rightarrow \infty} x(t)=0$,

(ii) if $r_{1}>r_{2}$, then $\lim _{t \rightarrow \infty} x(t)=+\infty$,

for all $t \in[-\tau, 0]$.

\section{Main Results}

In this section, for model (2), we aim to propose the sufficient conditions to guarantee the existence of the disease-free periodic solutions, the global stability of disease-free periodic solution, and the uniform persistence of the considered model.

3.1. Existence of the Disease-Free Periodic Solutions. Firstly, the analysis result is given to ensure the existence of the disease-free periodic solutions.
Theorem 3. If $\theta \mu /\left(1-e^{-\mu T}\right)<1$, (10) has a unique and positive periodic solution $\left(S^{*}(t), N^{*}(t)\right)$. Moreover (5) has a unique disease-free periodic solution $\left(S^{*}(t), 0,0, N^{*}(t)\right)$.

Proof. The existence of the disease-free periodic solution means that the number of sick people is zero, that is $I(t)=0$ for all $t \geq 0$. Thus (5) is transformed into

$$
\begin{gathered}
S^{\prime}(t)=A-\mu S(t), \\
E^{\prime}(t)=-\mu E(t), \\
N^{\prime}(t)=A-\mu N(t), \\
t \neq n T, \\
S\left(t^{+}\right)=S(t)-\theta \mu N(t), \\
E\left(t^{+}\right)=E(t), \\
N\left(t^{+}\right)=N(t), \\
t=n T .
\end{gathered}
$$

Noticing that $E(t)$ only appears in the second equation of (9), so we only need to consider the first and third equations of (9),

$$
\begin{gathered}
S^{\prime}(t)=A-\mu S(t), \\
N^{\prime}(t)=A-\mu N(t), \\
t \neq n T, \\
S\left(t^{+}\right)=S(t)-\theta \mu N(t), \\
N\left(t^{+}\right)=N(t), \\
t=n T .
\end{gathered}
$$

Let $N(n T)$ and $S(n T)$ represent the initial value of $N(t)$ and $S(t)$, at the time $t=n T$, respectively. For brevity, denote $N_{n}=N(n T), S_{n}=S(n T)$. Then, we can be integral in the pulse interval $[n T,(n+1) T]$, respectively, and for the total population and infected people, and we have

$$
\begin{aligned}
S(t) & =\frac{A}{\mu}-\left(\frac{A}{\mu}-S(n T)\right) e^{-\mu(t-n T)}, \\
N(t) & =\frac{A}{\mu}-\left(\frac{A}{\mu}-N(n T)\right) e^{-\mu(t-n T)} .
\end{aligned}
$$

According to (11), we have the following stroboscopic map:

$$
\left(\begin{array}{c}
S_{n+1} \\
N_{n+1}
\end{array}\right)=\left(\begin{array}{cc}
e^{-\mu T} & -\theta \mu \\
0 & e^{-\mu T}
\end{array}\right)\left(\begin{array}{c}
S_{n} \\
N_{n}
\end{array}\right)+\left(\begin{array}{l}
\frac{A}{\mu}\left(1-e^{-\mu T}\right) \\
\frac{A}{\mu}\left(1-e^{-\mu T}\right)
\end{array}\right)
$$

The Jacobi matrix of (12) is

$$
J=\left(\begin{array}{cc}
e^{-\mu T} & -\theta \mu e^{-\mu T} \\
0 & e^{-\mu T}
\end{array}\right) .
$$


The matrix $J$ has the characteristic roots $\lambda_{1}=\lambda_{2}=e^{-\mu T}$ with $\left|\lambda_{i}\right|=e^{-\mu t}<1(i=1,2)$. If $\theta \mu /\left(1-e^{-\mu T}\right)<1$, that is, $(A / \mu)-$ $\left(\theta A /\left(1-e^{-\mu T}\right)\right)>0$, then map (12) has a unique and positive fixed point $\left(S^{*}, N^{*}\right)$ with $S^{*}=(A / \mu)-\left(\theta A /\left(1-e^{-\mu T}\right)\right), N^{*}=$ $A / \mu$. Therefore, the periodic solution of (10) is

$$
\begin{array}{cc}
S^{*}(t)=\left\{\begin{array}{cc}
\frac{A}{\mu}-\frac{\theta A}{1-e^{-\mu T}} e^{-\mu(t-n T)}, & t \neq n T, \\
\frac{A}{\mu}-\frac{\theta A}{1-e^{-\mu T}}, & t=n T,
\end{array}\right. \\
N^{*}(t)=\frac{A}{\mu} .
\end{array}
$$

By using the second and fifth equations of (9), we have $\lim _{t \rightarrow \infty} E^{*}(t)=0$. Thus the proof of this theorem is complete.

3.2. Global Stability of Disease-Free Periodic Solution. In this subsection, the global stability of the disease-free periodic solution is discussed and the sufficient condition is given accordingly.

Theorem 4. If $R_{1}=\max \left\{R_{1}, \theta \mu /\left(1-e^{-\mu t}\right)\right\}<1$, then the disease-free periodic solution $\left(S^{*}(t), 0,0, A / \mu\right)$ of $(9)$ is globally attractive, where

$$
R_{1}=\frac{\beta\left((A / \mu)-\left(\theta \mu A /(\mu+\alpha)\left(1-e^{-\mu T}\right)\right)\right)+q \mu}{e^{\mu \tau}(\gamma+\alpha+\mu)} .
$$

Proof. By the first and fifth equations of (5) and $N^{\prime}(t) \geq A-$ $(\mu+\alpha) N(t)$, we have $N(t) \geq A /(\mu+\alpha)$, and then we have

$$
\begin{gathered}
S^{\prime}(t) \leq A-\mu S(t), \quad t \neq n T, n \in N \\
S\left(t^{+}\right) \leq S(t)-\frac{\theta \mu A}{\mu+\alpha}, \quad t=n T, n \in N .
\end{gathered}
$$

By Lemma 1, we obtain

$$
\begin{aligned}
S(t) \leq & S\left(0^{+}\right) \prod_{0<n T<t} e^{\int_{0}^{t}-\mu d s}+\sum_{0<n T<t} e^{\int_{n T}^{t}-\mu d s} \cdot\left(-\frac{\theta \mu A}{\mu+\alpha}\right) \\
& +\int_{0}^{t} \prod_{s<n T<t} e^{\int_{s}^{t}-\mu d \theta} \cdot A d s \\
\leq & S\left(0^{+}\right) e^{-\mu t}+\left(-\frac{\theta \mu A}{\mu+\alpha}\right) e^{-\mu t} \frac{e^{\mu T}\left(1-e^{\mu[t / T T T}\right)}{1-e^{\mu T}}+\bar{\Delta}
\end{aligned}
$$

where

$$
\begin{aligned}
\bar{\Delta} & =\int_{0}^{t} \prod_{s<n T<t} e^{\int_{s}^{t}-\mu d \theta} \cdot A d s \\
& =\frac{A}{\mu} e^{-\mu t} \int_{0}^{t} \prod_{s<n T<t} e^{\mu s} d \mu s \\
& =\frac{A}{\mu} e^{-\mu t} \int_{0}^{t / T} \prod_{\xi<n<t / T} e^{\mu T \xi} d \mu T \xi \\
& =\frac{A}{\mu} e^{-\mu t}\left[\int_{0}^{1} \prod_{\xi<n<t / T} e^{\mu T \xi} d \mu T \xi+\int_{1}^{2} \prod_{\xi<n<t / T} e^{\mu T \xi} d \mu T \xi\right. \\
& =\frac{A}{\mu} e^{-\mu t}\left[\frac{\left(e^{\mu T}-1\right)\left(1-e^{\mu T[t / T]}\right)}{1-e^{\mu T}}+e_{[t / T]} \prod_{\xi<n<t / T} e^{\mu T \xi} d \mu T \xi\right] \\
& =\frac{A}{\mu}-\frac{A}{\mu} e^{-\mu t} .
\end{aligned}
$$

So

$$
\begin{aligned}
S(t) \leq & S\left(0^{+}\right) e^{-\mu t}+\left(-\frac{\theta \mu A}{\mu+\alpha}\right) e^{-\mu t} \frac{e^{\mu T}\left(1-e^{\mu[t / T] T}\right)}{1-e^{\mu T}} \\
& +\frac{A}{\mu}-\frac{A}{\mu} e^{-\mu t} \\
\leq & e^{-\mu t}\left[S\left(0^{+}\right)-\frac{A}{\mu}+\frac{\theta \mu A}{(\mu+\alpha)\left(1-e^{-\mu T}\right)}\right] \\
& +\frac{A}{\mu}+\frac{\theta \mu A e^{\mu T([t / T]+1-(t / T))}}{(\mu+\alpha)\left(e^{\mu T}-1\right)} \\
\leq & e^{-\mu t}\left[S\left(0^{+}\right)-\frac{A}{\mu}+\frac{\theta \mu A}{(\mu+\alpha)\left(1-e^{-\mu T}\right)}\right] \\
& +\frac{A}{\mu}-\frac{\theta \mu A}{(\mu+\alpha)\left(1-e^{-\mu T}\right)},
\end{aligned}
$$

that is,

$$
\limsup _{t \rightarrow \infty} S(t) \leq \frac{A}{\mu}-\frac{\mu \theta A}{(\mu+\alpha)\left(1-e^{-\mu T}\right)} .
$$

Noticing $\theta \mu /\left(1-e^{-\mu T}\right)<1$, we have $\theta \mu /\left(1-e^{-\mu T}\right) \cdot \mu /(\mu+\alpha)<$ 1. Moreover, we obtain $(A / \mu)-\left(\mu \theta A /(\mu+\alpha)\left(1-e^{-\mu T}\right)\right)>0$. Then, for all $\varepsilon>0$, there exists $n_{1} \in N$ such that

$$
S(t) \leq \frac{A}{\mu}-\frac{\mu \theta A}{(\mu+\alpha)\left(1-e^{-\mu T}\right)}+\varepsilon \triangleq \eta
$$

for all $t \geq n_{1} T$. 
Subsequently, it follows from (21) and the third equation of (5) that

$$
\begin{aligned}
I^{\prime}(t) \leq & \frac{\beta \eta I(t-\tau)}{1+m I^{h}(t-\tau)} e^{-\mu \tau} \\
& +q \mu I(t-\tau) e^{-\mu \tau}-(\mu+\alpha+\gamma) I(t) \\
\leq & \beta \eta I(t-\tau) e^{-\mu \tau}+q \mu I(t-\tau) e^{-\mu \tau}-(\mu+\alpha+\gamma) I(t) \\
= & e^{-\mu \tau}(\beta \eta+q \mu) I(t-\tau)-(\mu+\alpha+\gamma) I(t)
\end{aligned}
$$

for $\forall t>n_{1} T+\tau$.

Consider the comparison system of (22)

$$
z^{\prime}(t)=e^{-\mu \tau}(\beta \eta+q \mu) z(t-\tau)-(\mu+\alpha+\gamma) z(t) .
$$

Due to $R_{1}<1+\varepsilon$, the following inequalities hold:

$$
\begin{gathered}
e^{-\mu \tau}\left[\beta\left(\frac{A}{\mu}-\frac{\theta \mu A}{(\mu+\alpha)\left(1-e^{-\mu T}\right)}+\varepsilon\right)+q \mu\right] \\
-(\gamma+\alpha+\mu)<0 \\
e^{-\mu \tau}(\beta \eta+q \mu)<(\gamma+\alpha+\mu)
\end{gathered}
$$

for $\forall \varepsilon>0$. By Lemma 2, we have $\lim _{t \rightarrow \infty} z(t)=0$. Note that $I(s)=z(s)=\varphi_{2}(s)>0$ for all $s \in[-\tau, 0]$. Then, according to the comparison theorem of differential equation, one gets $\lim _{t \rightarrow \infty} I(t)=0$.

Without loss of generality, assuming that there exists $t_{0}>$ 0 , we have $0<I(t)<\varepsilon<A / \alpha$ for all $t \geq t_{0}$. By the first and the fifth equations of (5), we have

$$
\begin{gathered}
S^{\prime}(t) \geq(A-q \mu \varepsilon)-(\beta \varepsilon+\mu) S(t), \quad t \neq n T, n \in N \\
S\left(t^{+}\right) \geq S(t)-\theta A, \quad t=n T, n \in N
\end{gathered}
$$

Considering the comparison systems of (25),

$$
\begin{gathered}
z_{1}^{\prime}(t)=(A-q \mu \varepsilon)-(\beta \varepsilon+\mu) z_{1}(t), \\
t \neq n T, n \in N, \\
z_{1}\left(t^{+}\right)=z_{1}(t)-\theta A, \quad t=n T, n \in N, \\
z_{1}\left(0^{+}\right)=S\left(0^{+}\right)
\end{gathered}
$$

when $n T<t \leq(n+1) T$, we obtain

$$
z_{1}^{*}(t)= \begin{cases}\frac{A-q \mu \varepsilon}{\beta \varepsilon+\mu}-\frac{\theta A}{1-e^{-(\beta \varepsilon+\mu) T}} e^{-(\beta \varepsilon+\mu)(t-n T)}, & t \neq n T \\ \frac{A-q \mu \varepsilon}{\beta \varepsilon+\mu}-\frac{\theta A}{1-e^{-(\beta \varepsilon+\mu) T}}, & t=n T,\end{cases}
$$

where

$$
\lim _{\varepsilon \rightarrow 0} z_{1}^{*}(t)=S^{*}(t)
$$

By the comparison theorem of impulsive differential equation, for $\forall \varepsilon_{1}>0$, there exists $T_{1}>t_{0}$, when $t>T_{1}$, and we obtain

$$
S(t)>z_{1}^{*}(t)-\varepsilon_{1}
$$

for all $t>T_{1}$. As $A-\mu N-\alpha \varepsilon \leq N^{\prime}(t), t \geq t_{0}, N(t) \geq$ $(A-\alpha \varepsilon) / \mu>0$. Then due to the first and the fifth equations of (5), we obtain

$$
\begin{gathered}
S^{\prime}(t) \leq A-\mu S(t), \quad t \neq n T, n \in N, \\
S\left(t^{+}\right) \leq S(t)-\theta A+(\alpha+\mu q) \theta \varepsilon, \quad t=n T, n \in N .
\end{gathered}
$$

Considering the comparison systems of (30),

$$
\begin{gathered}
z_{2}^{\prime}(t)=A-\mu z_{2}(t), \quad t \neq n T, n \in N, \\
z_{2}\left(t^{+}\right)=z_{2}(t)-\theta A+(\alpha+\mu q) \theta \varepsilon, \quad t=n T, n \in N, \\
z_{2}\left(0^{+}\right)=S\left(0^{+}\right),
\end{gathered}
$$

we have

$$
z_{2}^{*}(t)= \begin{cases}\frac{A}{\mu}-\frac{\theta A-(\alpha+\mu q) \theta \varepsilon}{1-e^{-\mu T}} e^{-\mu(t-n T)}, & t \neq n T \\ \frac{A}{\mu}-\frac{\theta A-(\alpha+\mu q) \theta \varepsilon}{1-e^{-\mu T}}, & t=n T\end{cases}
$$

when $n T<t \leq(n+1) T$.

Similarly, for $\forall \varepsilon_{1}>0$, there exists $T_{2}>t_{0}$ such that

$$
S(t)<\overline{z_{2}}(t)+\varepsilon_{1}
$$

for $t>T_{2}$. Denote $\bar{T}=\max \left\{T_{1}, T_{2}\right\}$. When $t>\bar{T}$, let $\varepsilon_{1} \rightarrow 0$. Then it follows from (29) and (33) that $S^{*}(t)-\varepsilon_{1}<S(t)<$ $S^{*}(t)+\varepsilon_{1}$, that is,

$$
\lim _{t \rightarrow \infty} S(t)=S^{*}(t)
$$

By substituting $I(t)=0$ into (5), we have $\lim _{t \rightarrow \infty} E(t)=0$, $\lim _{t \rightarrow \infty} N(t)=A / \mu$. Then, the proof of this theorem is complete.

3.3. Uniform Persistence of the Model. In this subsection, the definition of the uniform persistence is first given. Then the sufficient condition is proposed to ensure the uniform persistence of the addressed model.

Definition 5. If there exists a compact set $D \subset \Omega$, such that (2) at any periodic solution enters into $D$ and finally remains $D$ under the initial condition of (3), then (2) is uniformly persistent.

According to the above definition, we aim to present the analysis result about the uniform persistence for model (2). 
Theorem 6. If $R_{2}=\min \left\{R_{2}, R_{3}, R_{4}\right\}>1$ then the (2) is uniformly persistent, where

$$
\begin{gathered}
R_{2}=\frac{(A+q \mu)\left(1-e^{-\mu T}\right)+\theta A(\mu+\beta)-\left(1+m \delta^{h}\right)(\alpha+\gamma+\xi)}{q \mu\left(1-e^{-\mu T}\right)+\theta A \beta}, \\
R_{3}=\frac{(1-q)\left(1-e^{-(\delta \beta+\mu) T}\right)}{(\delta \beta+\mu) \theta}, \\
\delta=\frac{A}{\mu}, \quad \xi=\mu\left(1-q e^{-\mu \tau}\right) .
\end{gathered}
$$

Proof. It follows from $\Omega$ that, we obtain $N(t) \leq A / \mu=\delta$, then the solutions of (2) have upper bound. So we only need to consider the lower bound solution of (2).

First, we show the existence of a lower bound for $I(t)$. The third equation of (2) transforms the form as follows:

$$
\begin{aligned}
I^{\prime}(t)= & {\left[\frac{\beta S(t)}{1+m I^{h}(t)} e^{-\mu \tau}-(\alpha+\gamma+\xi)\right] I(t) } \\
& -e^{-\mu \tau} \frac{d}{d t} \int_{t-\tau}^{t}\left(\frac{\beta S(\theta)}{1+m I^{h}(\theta)}+q \mu\right) I(\theta) d \theta .
\end{aligned}
$$

Construct $V(t)=I(t)+e^{-\mu \tau} \int_{t-\tau}^{t}\left(\left(\beta S(\theta) /\left(1+m I^{h}(\theta)\right)\right)+\right.$ $q \mu) I(\theta) d \theta$. So $V(t)$ is a bounded function. Along the solution of (2), we have

$$
\begin{aligned}
& V^{\prime}(t) \\
& \quad=I^{\prime}(t)+e^{-\mu \tau} \frac{d}{d t} \int_{t-\tau}^{t}\left(\frac{\beta S(\theta)}{1+m I^{h}(\theta)}+q \mu\right) I(\theta) d \theta \\
& \quad=(\alpha+\gamma+\xi)\left[\frac{\beta S(t)}{1+m I^{h}(t)} e^{-\mu \tau} \cdot \frac{1}{\alpha+\gamma+\xi}-1\right] I(t) .
\end{aligned}
$$

By $R_{2}>1$, there exists $m_{2}^{*}=R_{2}-1>0$ satisfying

$$
m_{2}^{*}=\frac{\beta e^{-\mu \tau} A\left[1-e^{-\mu T}-\theta \mu\right]-\left(1+m \delta^{h}\right)(\alpha+\gamma+\xi)}{\left[\left(1-e^{-\mu T}\right) q \mu+\theta A \beta\right] \beta e^{-\mu \tau}}>0 .
$$

Then, we have

$$
\frac{\beta e^{-\mu \tau}}{\left(1+m \delta^{h}\right)(\alpha+\gamma+\xi)}\left[\frac{A-q \mu m_{2}^{*}}{\beta m_{2}^{*}+\mu}-\frac{\theta A}{1-e^{-\mu T}}\right]=1 .
$$

Subsequently, there exists $\varepsilon_{1}>0$, such that

$$
\frac{\beta e^{-\mu \tau}}{\left(1+m \delta^{h}\right)(\alpha+\gamma+\xi)}\left[\frac{A-q \mu m_{2}^{*}}{\beta m_{2}^{*}+\mu}-\frac{\theta A}{1-e^{-\left(\beta m_{2}^{*}+\mu\right) T}}-\varepsilon_{1}\right]>1 .
$$

So

$$
\frac{\beta e^{-\mu \tau}}{\left(1+m \delta^{h}\right)(\alpha+\gamma+\xi)} \Delta>1
$$

where

$$
\Delta=\frac{A-q \mu m_{2}^{*}}{\beta m_{2}^{*}+\mu}-\frac{\theta A}{1-e^{-\left(\beta m_{2}^{*}+\mu\right) T}}-\varepsilon_{1} .
$$

Now we prove that $I(t) \leq m_{2}^{*}$ is not true. Otherwise, assume that there exists $t_{0}>0$, and when $t>t_{0}$, there is $I(t)<m_{2}^{*}$. By using the first and the fifth equations of (2), we have

$$
\begin{gathered}
S^{\prime}(t) \geq\left(A-q \mu m_{2}^{*}\right)-\left(\beta m_{2}^{*}+\mu\right) S(t), \\
t \neq n T, n \in N, \\
S(t) \geq S(t)-\theta A, \quad t=n T, n \in N .
\end{gathered}
$$

By Lemma 1, there exists $T_{1} \geq t_{0}+\tau$, and we have

$$
S(t)>\frac{A-q \mu m_{2}^{*}}{\beta m_{2}^{*}+\mu}-\frac{\theta A}{1-e^{-\left(\beta m_{2}^{*}+\mu\right) T}}-\varepsilon_{1} \triangleq \Delta
$$

for $t>T_{1}$. Together with (37) and (44), one gets

$$
V^{\prime}(t)>(\gamma+\xi)\left[\frac{\beta e^{-\mu \tau}}{\left(1+m \delta^{h}\right)(\alpha+\gamma+\xi)} \cdot \Delta-1\right] I(t)
$$

Denote $I^{l}=\min _{t \in\left[T_{1}, T_{1}+\tau\right]} I(t)$. Then $t \geq T_{1}>t_{0}$, that is, $I(t) \geq$ $I^{l}$. Otherwise there exists $T_{2}>0$, such that $t \in\left[T_{1}, T_{1}+\tau+T_{2}\right]$. Then there are $I(t) \geq I^{l}, I\left(T_{1}+\tau+T_{2}\right)=I^{l}$, and $I^{\prime}\left(T_{1}+\tau+T_{2}\right) \leq$ 0 . It follows from the second equation of (2) and (41) that

$$
\begin{aligned}
& I^{\prime}\left(T_{1}+\tau+T_{2}\right) \\
& \quad \geq(\alpha+\gamma+\xi)\left[\frac{\beta e^{-\mu \tau}}{\left(1+m \delta^{h}\right)(\alpha+\gamma+\xi)} \Delta-1\right] I^{l}>0,
\end{aligned}
$$

which is a contradiction. So, for $t \geq T_{1}$, according to (36), $I(t) \geq I^{l}, V^{\prime}(t)>0$, that is $\lim _{t \rightarrow \infty} V(t)=+\infty$. This is, a contradiction of which $V(t)$ is bounded. So when $t>t_{0}$, $I(t)<m_{2}^{*}$ is not tenable.

Two cases to prove are given as follows.

Case 1. If, for $t$ which is sufficiently large, $I(t) \geq m_{2}^{*}$, hence the statements are proved.

Case 2. If $I(t)$ is shocked near $m_{2}^{*}$, let $m_{2}=\min \left\{m_{2}^{*} / 2\right.$, $\left.m_{2}^{*} e^{-(\alpha+\gamma+\mu) \tau}\right\}$. There exists two constants: $\bar{t}$ and $\omega$ satisfying $I(\bar{t})=I(\bar{t}+\omega)=m_{2}^{*}$, and when $\bar{t}<t<\bar{t}+\omega$, there is $I(t)<m_{2}^{*}$. Because $I(t)$ is a continuous and bounded function which is not affected by pulse, then $I(t)$ is uniformly continuous. So, there exists $0<T_{3}<\tau$ ( $T_{3}$ depends on the selection of $\bar{t}$ ), met for all $\bar{t}<t<\bar{t}+T_{3}$, and there is $I(t)>m_{2}^{*} / 2$. If $\omega<T_{3}$. The conclusion was established. If $T_{3}<\omega<\tau$, then, by the second equation of (5) that we have when $\bar{t}<t<\bar{t}+\Phi$, $I^{\prime}(t) \geq-(\alpha+\gamma+\mu) I(t)$; again by $I(\bar{t})=m_{2}^{*}$, there is $I(t) \geq m_{2}^{*} e^{-(\alpha+\gamma+\mu) \tau}$, and obviously $I(t) \geq m_{2}$ is established. Similarly, if $\omega \geq \tau$, then, by the second equation of (5), when $\bar{t}<t<\bar{t}+\tau, I(t) \geq m_{2}$. Then proof is as follows, when 
$\bar{t}+\tau \leq t \leq \bar{t}+\omega$, and there is still $I(t) \geq m_{2}$. If not, there exists $\bar{T} \geq 0$, when $\bar{t} \leq t \leq \bar{t}+\tau+\bar{T}$, and there are $I(t) \geq m_{2}$, $I(\bar{t}+\tau+\bar{T})=m_{2}$, and $I^{\prime}(\bar{t}+\tau+\bar{T}) \leq 0$. It follows from the second equation of (2) that

$$
I^{\prime}(\bar{t}+\tau+\bar{T}) \geq\left[\frac{\beta e^{-\mu \tau}}{\left(1+m \delta^{h}\right)(\alpha+\gamma+\xi)} \Delta-1\right] m_{2}>0 .
$$

It is a contradiction. So when $\bar{t}<t<\bar{t}+\emptyset$, there is $I(t) \geq m_{2}$.

Above all, any positive periodic solution of (2) which for sufficiently large $t$, it can be concluded that $I(t) \geq m_{2}$.

It follows from the first and the fifth equations of (2) that

$$
\begin{gathered}
S^{\prime}(t) \geq(1-q) A-(\delta \beta+\mu) S(t), \quad t \neq n T, n \in N, \\
S\left(t^{+}\right) \geq S(t)-\theta A, \quad t=n T, n \in N .
\end{gathered}
$$

It follows from Lemma 1 that

$$
\begin{aligned}
S(t) \geq & e^{-(\delta \beta+\mu) t}\left[S\left(0^{+}\right)-\frac{(1-q) A}{\delta \beta+\mu}+\frac{\theta A}{1-e^{-(\delta \beta+\mu) T}}\right] \\
& +\frac{(1-q) A}{\delta \beta+\mu}-\frac{\theta A e^{(\delta \beta+\mu)(T[t / T]+T-t)}}{e^{(\delta \beta+\mu) T}-1} .
\end{aligned}
$$

Since $0<T[t / T]+T-t<T$, so

$$
\begin{aligned}
S(t) \geq & e^{-(\delta \beta+\mu) t}\left[S\left(0^{+}\right)-\frac{(1-q) A}{\delta \beta+\mu}+\frac{\theta A}{1-e^{-(\delta \beta+\mu) T}}\right] \\
& +\frac{(1-q) A}{\delta \beta+\mu}-\frac{\theta A}{1-e^{-(\delta \beta+\mu) T}} .
\end{aligned}
$$

Thus $\lim _{t \rightarrow \infty} S(t) \geq A\left[((1-q) /(\delta \beta+\mu))-\left(\theta /\left(1-e^{-(\delta \beta+\mu) T}\right)\right)\right]$; it follows from $R_{3}>1$ that $((1-q) /(\delta \beta+\mu))-(\theta /(1-$ $\left.\left.e^{-(\delta \beta+\mu) T}\right)\right)>0$, that is; for $\forall \varepsilon>0$, there exists $T_{4}>0$, when $t>T_{4}$, and there is

$$
S(t) \geq A\left[\frac{1-q}{\delta \beta+\mu}-\frac{\theta}{1-e^{-(\delta \beta+\mu) T}}\right]-\varepsilon \triangleq m_{1} .
$$

Because of the second equation of (2)

$$
\begin{aligned}
E(t) & =\int_{t-\tau}^{t} \frac{\beta S(u) I(u)}{1+m I^{h}(u)} e^{-\mu(t-u)} d u+\int_{t-\tau}^{t} q \mu I(u) e^{-\mu(t-u)} d u \\
& \geq\left(\frac{\beta m_{1} m_{2}}{\mu\left(1+m \delta^{h}\right)}+q m_{2}\right)\left(1-e^{-\mu \tau}\right) \triangleq m_{3} .
\end{aligned}
$$
that

Subsequently, it follows from the fourth equation of (2)

$$
R^{\prime}(t) \geq r m_{2}-\mu R(t) .
$$

Similarly, there is $R(t) \geq r m_{2} / \mu \triangleq m_{4}$ satisfying

$$
\begin{gathered}
\bar{D}=\left\{(S, E, I, R) \mid m_{1} \leq S(t) \leq \delta, m_{2} \leq I(t) \leq \delta,\right. \\
\left.m_{3} \leq E(t) \leq \delta, m_{4} \leq R(t) \leq \delta\right\} .
\end{gathered}
$$

Then, $\bar{D}$ is a bounded compact set, nontrivial for any cycle solution of (2) to enter and stay in $\bar{D}$ inside. Hence, it completes the proof of this theorem.

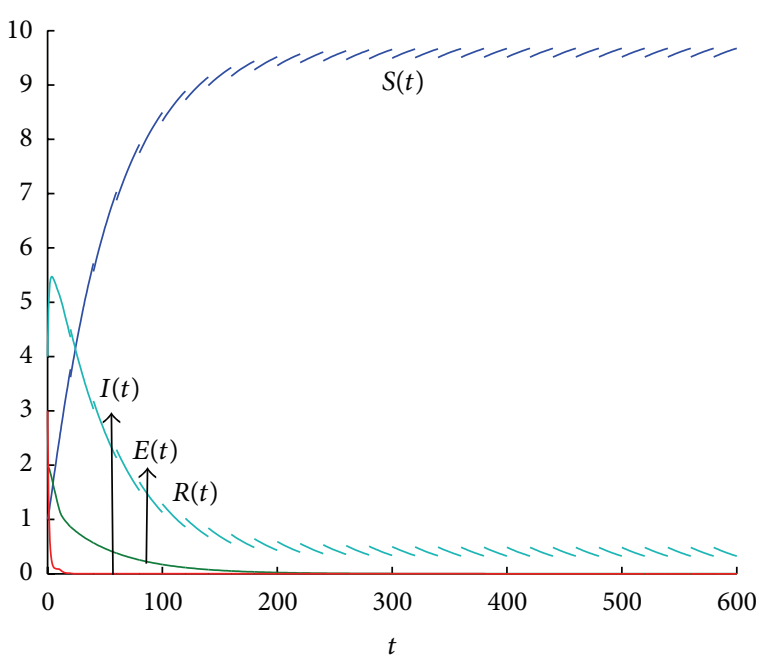

FIgURE 1: The global attractability of disease-free periodic solution.

Remark 7. Up till now, we investigate the delay epidemic model with vertical transmission, constant input and nonlinear incidence. The sufficient conditions are given to guarantee the existence of the disease-free periodic solutions, the global stability of disease-free periodic solution, and the uniform persistence of the considered model. It is worth mentioning that, according to the statistics of the suspected patients and patients and using the data identification approach, the parameters in the model can be determined when the disease outbreaks. Subsequently, the illness trend of the epidemic can be predicted. Hence, we can make the reasonable control measures. One of the future research directions would be to apply the developed results to make the control strategy by properly considering the real information on the epidemic data.

\section{A Numerical Simulation}

For comparisons, we consider the following five cases.

Case 1. Let the parameters be specified as certain fixed values in (2): $A=0.2, \mu=0.02, \beta=0.05, q=0.8, \gamma=0.5$, $\alpha=0.3, \theta=0.8, \tau=10, T=20$, and $m=h=1$; then the result $R_{1}=\max \{0.5137,0.04853\}<1$ is obtained after calculation. By Theorem 4, we know that the diseasefree periodic solution of (2) is globally attractive. Setting the initial value $S(0)=1, E(0)=2, I(0)=3$, and $R(0)=4$, the numerical simulation of the diagram is shown in Figure 1.

Case 2. Take the following parameters: $\tau=1, \alpha=0.01, A=$ $0.5, \beta=0.12, \mu=0.1, q=0.8, m=0.02, h=0.8, \gamma=0.06$, $T=10, p=1-q$, and $\theta=0.1$; we calculate that $R_{2}=$ $\min \{5.2830,2.8545\}>1$. By Theorem 6, (2) is uniform persistence. Letting the initial $S(0)=0.5, E(0)=1, I(0)=$ $1.5, R(0)=2$, the numerical simulation of (2) is shown in Figure 2.

Case 3. The parameter of (2) is the same as that in Figure 1 except for parameter $\beta$. Taking $\beta=0.08$, we have 


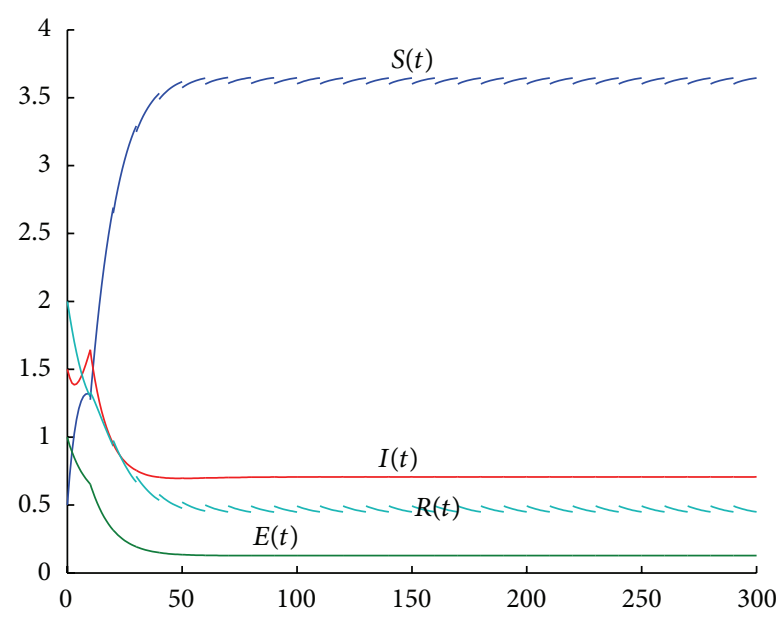

Figure 2: The uniform persistence of disease when $\beta=0.12, q=0.8$, and $\theta=0.1$.

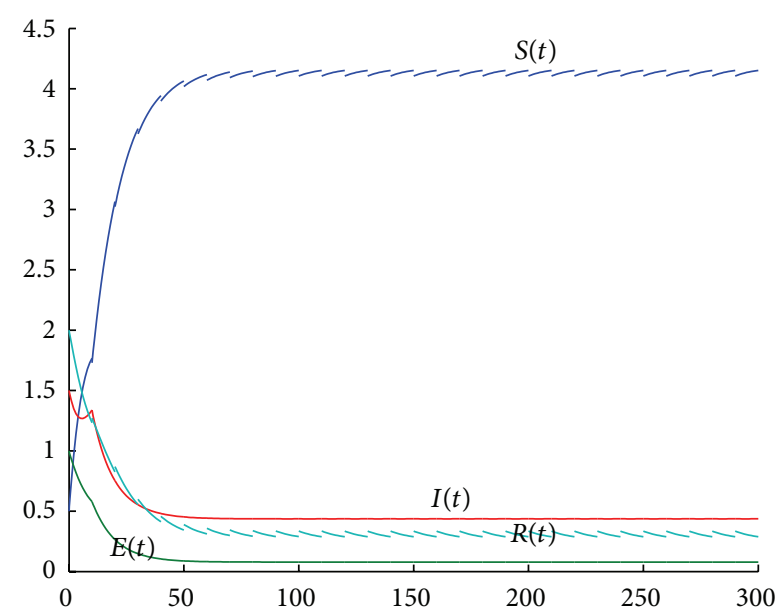

FIGURE 3: The uniform persistence of disease when $\beta=0.08$.

$R_{2}=\min \{5.4400,3.9730\}>1$. Then, by Theorem $6,(2)$ is of uniform persistence. The corresponding numerical simulation of (2) is shown in Figure 3.

Case 4. If the parameter of (2) is the same as in Figure 1 except $q$. Taking $q=0.1$, we obtain $R_{2}=\min \{20.1653,12.8454\}>1$. By Theorem 6, (2) is of uniform persistence. Accordingly, the simulation of (2) is shown in Figure 4.

Case 5. If the parameter of (2) is the same as in Figure 1 except $\theta$. Taking $\theta=0.9$, we have $R_{2}=\min \{4.9835,1.2496\}>1$. By Theorem 6, (2) is of uniform persistence. The numerical simulation of (2) is shown in Figure 5.

It can be seen that $I(t)$ is more influenced by the change of $\beta, p$ but is less influenced by the change of $\theta$.

By Figures 2 and 3, we can see that when the contact rate is smaller, the numbers of lurker and infective are reduced, but the numbers of susceptible and removed accordingly changed much. Also, there is a proportional relationship between the

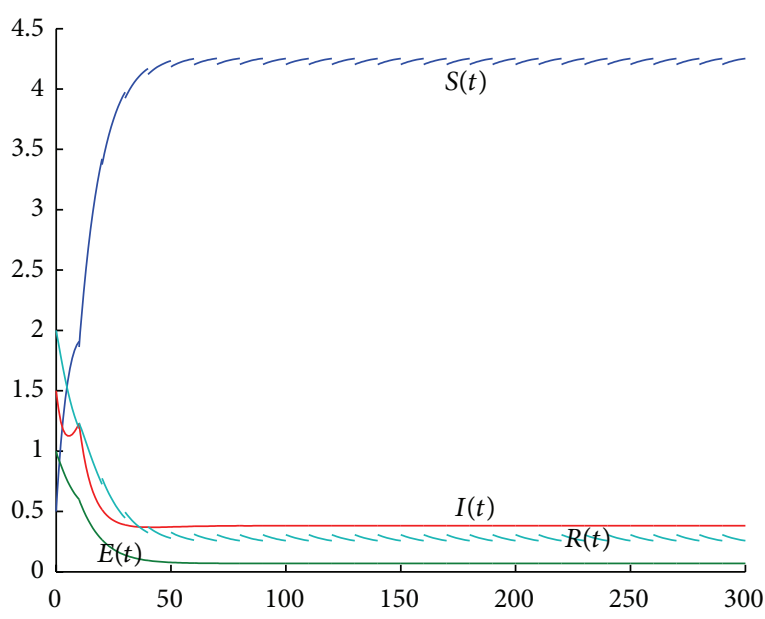

FIgURE 4: The uniform persistence of disease when $q=0.1$.

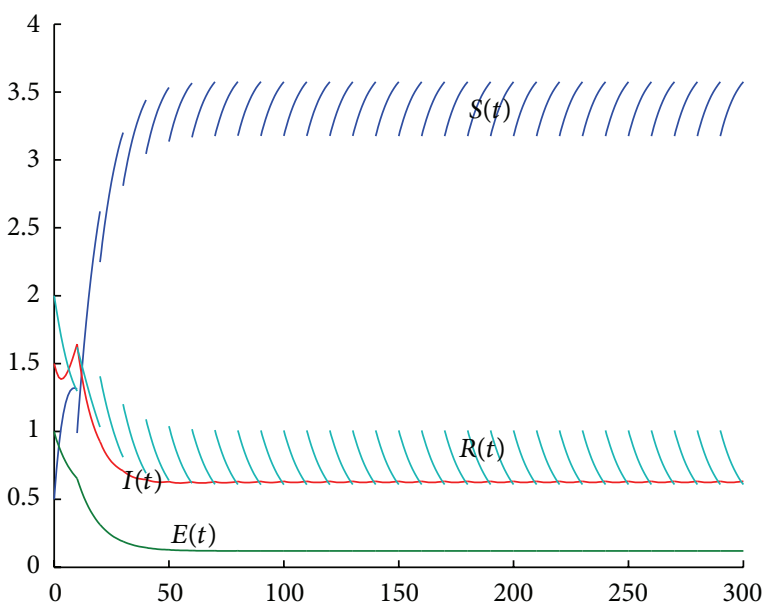

FIgURE 5: The uniform persistence of disease when $\theta=0.9$.

size of the extent of epidemic and the contact rate. By comparing Figures 2 and 4, when the vertical transmission rate is bigger, the number of infective is increased by controlling the vertical transmission rate of infected newborn. Moreover, by comparing Figures 2 and 5, the increase of vaccination rate has a certain effect for the control of the disease, but it is not obvious. Therefore, if we reduce the extent of the epidemic, the measure of one is reducing the contact rate and vertical transmission rate. From the simulation the feasibility and usefulness of the proposed main results are confirmed.

\section{Conclusions}

In this paper, we have discussed the SEIR model with the pulse vaccination, the constant input item of population, and the vertical transmission. By employing the impulsive differential inequality and the stroboscopic map, the existence conditions of the disease-free periodic solution of the model have been given. Also, the sufficient conditions of globally attractive and uniform persistence have been proposed. Finally, a numerical example has been given to illustrate the 
validity of the proposed results. One of our future research interests is to extend the main results of the analysis and synthesis of gene regulatory networks or complex dynamical systems as discussed in [27-32].

\section{Acknowledgments}

This work was supported in part by the National Natural Science Foundation of Heilongjiang Province under Grant no. A200502 and the Foundation of Educational Commission of Heilongjiang Province under Grant no. 12521099.

\section{References}

[1] V. D. Mil'man and A. D. Myškis, "On the stability of motion in the presence of impulses," Sibirskii Matematicheskii Journal, vol. 1, no. 2, pp. 233-237, 1960 (Russian).

[2] A. N. Perestyuk and A. M. Samoilenko, "Stability of solutions of differential equations with impulse effect," Different Equation, vol. 13, no. 1, pp. 1981-1992, 1977.

[3] Z. Agur, L. Cojocaru, G. Mazor, R. M. Anderson, and Y. L. Danon, "Pulse mass measles vaccination across age cohorts," Proceedings of the National Academy of Sciences of the United States of America, vol. 90, no. 24, pp. 11698-11702, 1993.

[4] S. Gao, L. Chen, and Z. Teng, "Impulsive vaccination of an SEIRS model with time delay and varying total population size," Bulletin of Mathematical Biology, vol. 69, no. 2, pp. 731-745, 2007.

[5] H. Liu, J. Yu, and G. Zhu, "Global asymptotic stable eradication for the SIV epidemic model with impulsive vaccination and infection-age," Journal of Systems Science \& Complexity, vol. 19, no. 3, pp. 393-402, 2006.

[6] A. d'Onofrio, "Mixed pulse vaccination strategy in epidemic model with realistically distributed infectious and latent times," Applied Mathematics and Computation, vol. 151, no. 1, pp. 181187, 2004.

[7] X.-Z. Meng, L.-S. Chen, and Z.-T. Song, "Global dynamics behaviors for new delay SEIR epidemic disease model with vertical transmission and pulse vaccination," Applied Mathematics and Mechanics (English Edition), vol. 28, no. 9, pp. 1259-1271, 2007.

[8] A. d'Onofrio, "Stability properties of pulse vaccination strategy in SEIR epidemic model," Mathematical Biosciences, vol. 179, no. 1, pp. 57-72, 2002.

[9] J. Hu, Z. Wang, B. Shen, and H. Gao, "Gain-constrained recursive filtering with stochastic nonlinearities and probabilistic sensor delays," IEEE Transactions on Signal Processing, vol. 61, no. 5, pp. 1230-1238, 2013.

[10] J. Hu, Z. Wang, H. Gao, and L. K. Stergioulas, "Robust sliding mode control for discrete stochastic systems with mixed time delays, randomly occurring uncertainties, and randomly occurring nonlinearities," IEEE Transactions on Industrial Electronics, vol. 59, no. 7, pp. 3008-3015, 2012.

[11] J. Hu, Z. Wang, Y. Niu, and L. K. Stergioulas, " $H_{\infty}$ sliding mode observer design for a class of nonlinear discrete time-delay systems: a delay-fractioning approach," International Journal of Robust and Nonlinear Control, vol. 22, no. 16, pp. 1806-1826, 2012.

[12] J. Hu, Z. Wang, H. Gao, and L. K. Stergioulas, "Robust $H_{\infty}$ sliding mode control for discrete time-delay systems with stochastic nonlinearities," Journal of the Franklin Institute, vol. 349, no. 4, pp. 1459-1479, 2012.
[13] X.-B. Zhang, H.-F. Huo, X.-K. Sun, and Q. Fu, “The differential susceptibility SIR epidemic model with time delay and pulse vaccination," Journal of Applied Mathematics and Computing, vol. 34, no. 1-2, pp. 287-298, 2010.

[14] Y. Du and R. Xu, "A delayed SIR epidemic model with nonlinear incidence rate and pulse vaccination," Journal of Applied Mathematics \& Informatics, vol. 28, no. 5-6, pp. 1089-1099, 2010.

[15] Z. Zhao, Z. Li, and L. Chen, "Existence and global stability of periodic solution for impulsive predator-prey model with diffusion and distributed delay," Journal of Applied Mathematics and Computing, vol. 33, no. 1-2, pp. 389-410, 2010.

[16] C. Huang and X. An, "An impulsive vaccination SEIRS epidemic model with multi-delay a nonlinear incidence rate," Journal of Lanzhou University of Technology, vol. 37, no. 1, pp. 121-125, 2011.

[17] Y. Du and R. Xu, "Pulse vaccination of a SEIRS epidemic model with time delays a nonlinear incidence rate," Journal of Beihua University (Natural Science), vol. 12, no. 3, pp. 258-264, 2011.

[18] S. J. Gao and Z. D. Teng, "Impulsive vaccination of an SIRS model with saturation infectious force and constant recruitment," Journal of Biomathematics, vol. 23, no. 2, pp. 209-217, 2008.

[19] C. Wei and L. Chen, "A delayed epidemic model with pulse vaccination," Discrete Dynamics in Nature and Society, vol. 2008, Article ID 746951, 12 pages, 2008.

[20] X. Meng, Z. Li, and X. Wang, "Dynamics of a novel nonlinear SIR model with double epidemic hypothesis and impulsive effects," Nonlinear Dynamics, vol. 59, no. 3, pp. 503-513, 2010.

[21] W. J. Xu, "The SIQRS epidemic model of impulsive vaccination with constant input and saturation incidence rate," Journal of Systems Science and Mathematical Sciences, vol. 30, no. 1, pp. 4352, 2010.

[22] X.-Z. Meng, L.-S. Chen, and Z.-T. Song, "Global dynamics behaviors for new delay SEIR epidemic disease model with vertical transmission and pulse vaccination," Applied Mathematics and Mechanics (English Edition), vol. 28, no. 9, pp. 1259-1271, 2007.

[23] Y. Z. Pei, S. Y. Liu, C. G. Li, and S. J. Gao, "A pulse vaccination epidemic model with multi-delay and vertical transmission," Chinese Annals of Mathematics A, vol. 30, no. 5, pp. 669-676, 2009.

[24] J. Zhu, W. Li, and L. Zhu, "An SIR epidemic model with birth pulse and pulse vaccination," Journal of Biomathematics, vol. 26, no. 3, pp. 490-496, 2011.

[25] V. Lakshmikantham, D. D. Baĭnov, and P. S. Simeonov, Theory of Impulsive Differential Equations, World Scientific Publishing, River Edge, NJ, USA, 1989.

[26] K. Yang, Delay Differential Equations with Applications in Population Dynamics, Academic Press, San Diego, Calif, USA, 1993.

[27] J. Hu, Z. Wang, H. Gao, and L. K. Stergioulas, "Extended Kalman filtering with stochastic nonlinearities and multiple missing measurements," Automatica, vol. 48, no. 9, pp. 20072015, 2012.

[28] Z. Wang, H. Wu, J. Liang, and X. Liu, "On modeling and state estimation for genetic regulatory networks with polytopic uncertainties," IEEE Transactions on NanoBioscience, vol. 12, no. 1, pp. 13-20, 2013.

[29] J. Hu, Z. Wang, H. Gao, and L. K. Stergioulas, "Probability-guaranteed $H_{\infty}$ finite-horizon filtering for a class of nonlinear timevarying systems with sensor saturations," Systems \& Control Letters, vol. 61, no. 4, pp. 477-484, 2012. 
[30] Z. Wang, Y. Wang, and Y. Liu, "Global synchronization for discrete-time stochastic complex networks with randomly occurred nonlinearities and mixed time delays," IEEE Transactions on Neural Networks, vol. 21, no. 1, pp. 11-25, 2010.

[31] J. Hu, Z. Wang, B. Shen, and H. Gao, "Quantised recursive filtering for a class of nonlinear systems with multiplicative noises and missing measurements," International Journal of Control, vol. 86, no. 4, pp. 650-663, 2013.

[32] Z. Wang, X. Liu, Y. Liu, J. Liang, and V. Vinciotti, “An extended kalman filtering approach to modeling nonlinear dynamic gene regulatory networks via short gene expression time series," IEEE/ACM Transactions on Computational Biology and Bioinformatics, vol. 6, no. 3, pp. 410-419, 2009. 


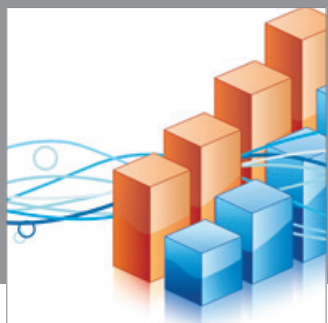

Advances in

Operations Research

mansans

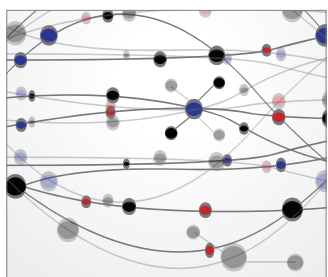

The Scientific World Journal
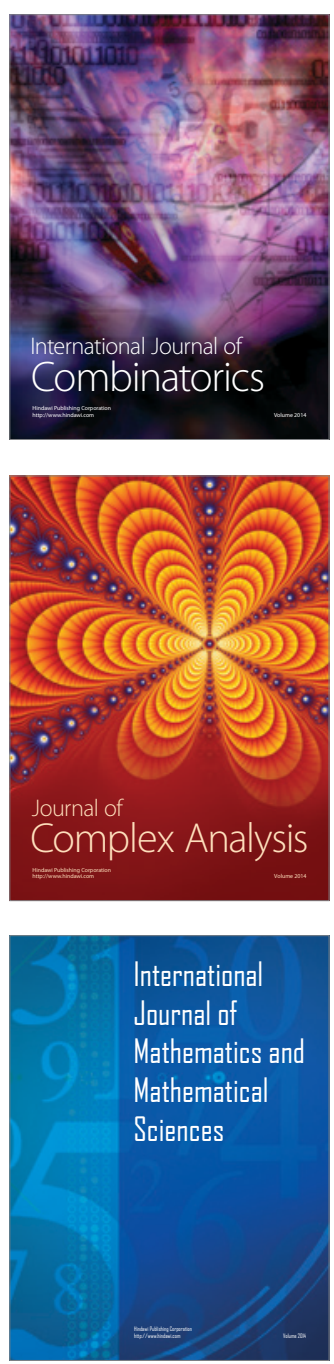
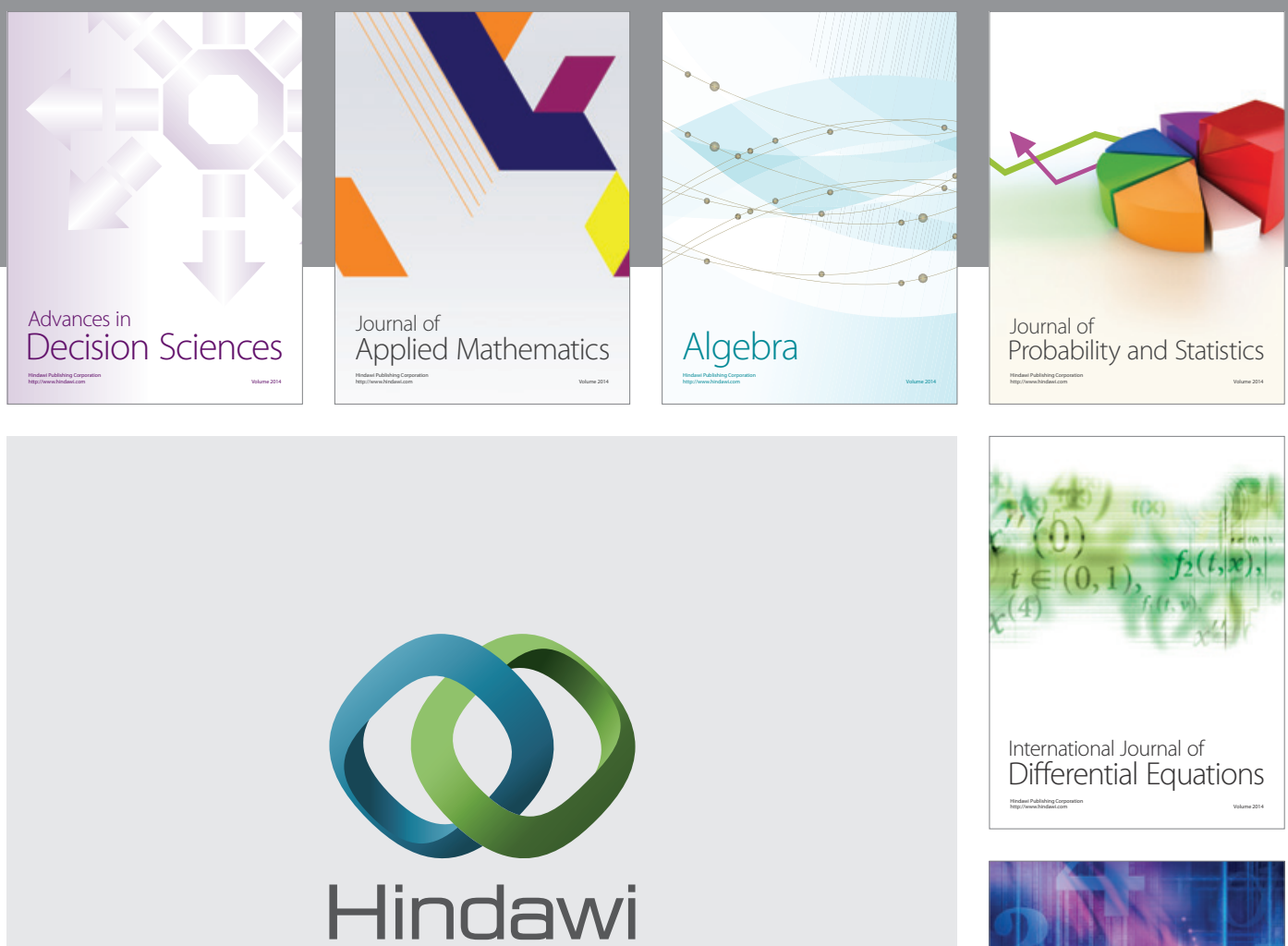

Submit your manuscripts at http://www.hindawi.com
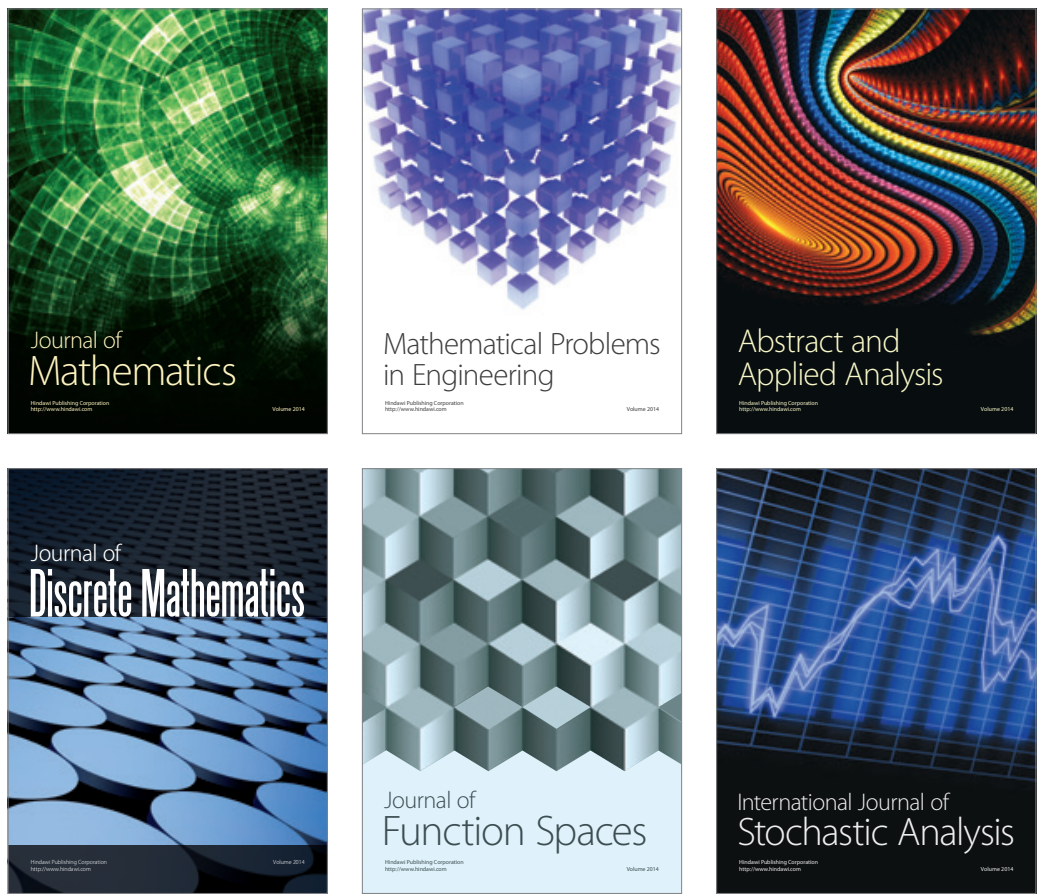

Journal of

Function Spaces

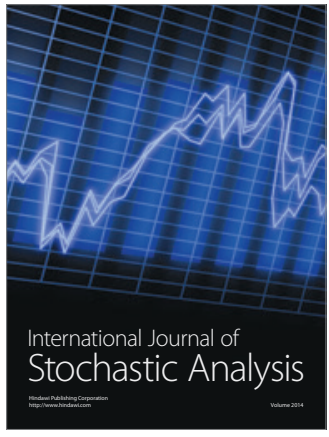

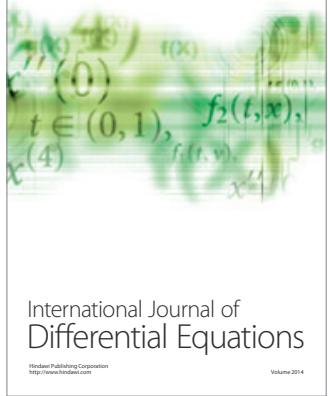
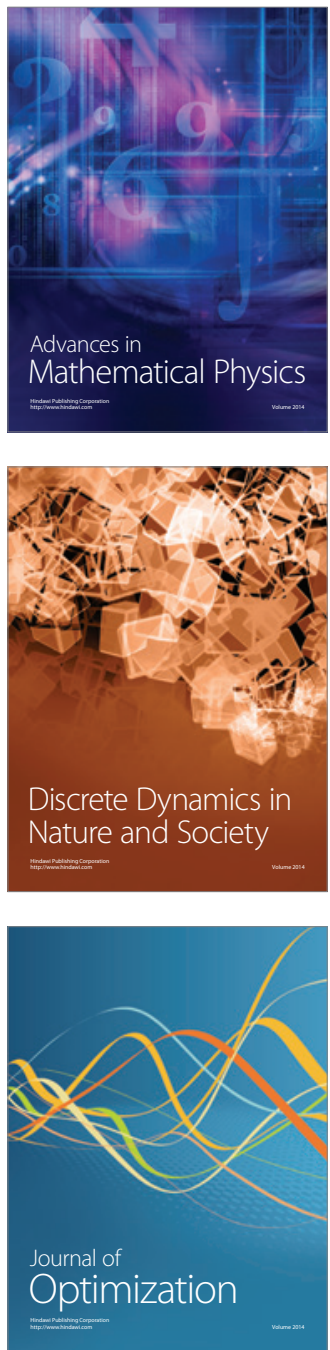